\title{
Efficient coordination in the lab
}

\begin{abstract}
In an infinitely repeated matching pennies game with asymmetric information, Gossner et al. (2006) studied coordination levels among agents and obtained that players may use a block codification using signaling mistakes in order to efficiently coordinate. Inspired in that model, we experimentally test coordination in the lab. We first model and establish the appropriate length of the sequence played by nature and the block strategy for a finitely repeated version of the game, where the majority rule with 3-length blocks results as the optimal block codification. Our experimental data give support to the main results of the original model with respect to the codification rule using signaling mistakes.
\end{abstract}

Keywords: coordination game, experiment, cheap-talk

JEL codes: D8, C91, C73

\section{Introduction}

Strategic information transmission is a process that plays a crucial role in many situations in which agents' decisions depend on disclosure information. In fact, the lack of information is one of main drawbacks to reach agreements. Hence, sharing information is a pivotal point that allows agents to get more profitable agreements. Furthermore, there exists a trade-off between revealed information and profit, which is due to strategic concerns. In Crawford and Sobel's (1982) words, revealing all information to the opponent is not usually the most advantageous policy. As Blume and Ortmann (2007) highlight, costless messages help overcome strategic uncertainty, problems equilibrium selection as well as coordination failure.

As a benchmark structure, information transmission between a sender and a receiver occurs when a message in a common language is sent through a transmission 
channel. Specifically, the sender is an agent with private information who sends a message revealing "some" information to the receiver, who takes a decision affecting both agents accordingly. The present work concerns strategic information transmission under asymmetric information.

In their seminal paper Crawford and Sobel (1982) introduced an one-sided communication model between an informed sender and an uninformed receiver and show how the conflict of interest has a negative effect on the flow of information. Based in that seminal model, there is a large number of applications of strategic information transmission. For example, applications to corporations (Watson, 1996; Kartik, 2005), to operation management (Allon and Bassamboo, 2011; Allon et al., 2011) or to political sciences (Gilligan and Krehbiel, 1989; Krishna and Morgan, 2001).

Some of our setting's features are in common with Crawford and Sobel's framework: 1) Information is transmitted in an one-sided communication channel. 2) The sender, player 1 , is a fully informed player who has complete and perfect information about nature. While the receiver, player 2 , is an uninformed player who knows about the no-player existence and actions in the past. 3) Sharing information is costless for the sender. 4) Decisions of the receiver have an effect on both players' payoffs.

Some other features are specific to our communication protocol, already introduced in Gossner et al. (2003): 5) The sender and receiver form a team with aligned interests. 6) Players play against the Nature modelized as an i.i.d. process. 7) The team and the Nature face decisions repeatedly. 8) The sender has private information on the future state of nature; while the receiver has public information about the history of nature's past states. 9) There is a positive gain when both players match nature's,

From a theoretical perspective, Gossner et al. (2003) characterize the equilibrium payoff that the team can both guarantee and defend against any behavior of the Nature. As it is already mentioned, the Nature behaves as an i.i.d. process, therefore the value (in correlated strategies) of the repeated zero sum game where the sender and the receiver play as a team against the Nature is related with their equilibrium payoff. Moreover, they construct equilibrium strategies of communication between the sender and the receiver in this infinitely repeated set-up based on block ${ }^{1}$ coding. To be precise, block coding strategies refer to the way how players communicate their following sequence of actions. Actually, the sequence of actions pairs are chopped in blocks and each block conveys information for the next block of actions pairs. The codification skills allow to the players to achieve the target payoff and to guarantee

\footnotetext{
${ }^{1}$ This technique is not new in the literature of repeated games under asymmetric information (AADIR BIBLIOGRAFIA).
} 
stability conditions. In this setup, the transmission information is only meanwhile the play, the sequence of actions played by the sender are encrypted working as messages, and the receiver decodes messages according to a common team's codebook.

The aim of this paper is to contrast whether under asymmetric uncertainty between players but still being dominant ${ }^{2}$ to transmit information, individuals actually implement such a kind of "block strategies". To do so, we have adapted the above situation to be properly contrasted in a controlled environment as a experimental laboratory. First, we provide a theoretical characterization of optimal block strategies considering that players interact a finite number of periods. Second, fixing the majority rule with blocks of size three, we establish the corresponding length of the game. We obtain the duration of 55 periods. These two features were the parameters used to run an experiment that fits the model and tests its robustness in the lab.

In our experiment, we implement a specific channel for communication between players. Before the game, a chat is activated during 3 minutes. In this time, players had the possibility to write free messages designing their strategies without explicit cost. This chat allowed to the players to fix the common ${ }^{3}$ codebook and the decodification rules that players may eventually perform. Once the chat was closed, the realizations of the 55 actions of the Nature were drawn following an i.i.d. process with law $\left(\frac{1}{2}, \frac{1}{2}\right)$. Player 1 was informed of all of them since in his/her screen a matrix of all realizations was active the whole sequence of actions. The play lates 55 stages and the information available for each player was different. At stage $t$, player 1 had in the screen the whole sequence of action of the Nature, his/her actions played so far and the sequence of action of the other player played until this stage. Nevertheless, player 2 had the whole history of the action triples of length $t-1$ : Nature, the other player and their own sequence of actions played until this stage. In all this phase, verbal communication was forbidden. Therefore, Player 1 that knew how the Nature plays in the future may signal to player 2 by using his/her own actions some event to reach better coordination. How much information was transmitted depends on how informative the signal of player 1 was and how receptive player 2 was in receiving the signal. This eventually determined the payoff of the two players as the number of concordance with the Nature's actions.

We analyse the effectiveness of the chat in transmitting information in the terms of the theoretical model. That is, without specifically analysing what the messages in the chat contain, we test whether and how much do players coordinate under such

\footnotetext{
${ }^{2}$ Given that there is a positive gain when both players match nature's, the sender has an incentive to share information in order to improve his own gains. Thus, actions' coordination is possible being a strictly dominant strategy for both players.

${ }^{3}$ This phase fits the assumption in Gossner et al. (2003) of the common set of strategies.
} 
conditions and, therefore, whether the model predicts reasonable strategies that could be observed from real heterogeneous agents.

Among our main experimental results we find that subjects design strategies at three levels of coordination. First, strategies at low level where the receiver ignores or misunderstands the sender's message, coordinating actions by pure chance. Second, a medium level of coordination in which strategies transmit successfully information by following a joint coordination code. A third level of coordination, the richest, where coordination codes achieve payoffs close to the optimal predicted by the theoretical model.

Additionally, we estimate the theoretical model with the data obtained in the experiment and find that actions by the receiver are significantly explained by actions played by the nature and the sender. With respect to senders, their signals are precise most of the times.

The paper is structured as follows. Section 2 reviews related literature on strategic information transmission. In Section 3 we describe the game and the theoretical framework. Section 4 explains in detail the experimental environment. In Section 5 we describe the data analysis and highlight the main results. Section 6 concludes.

\section{Related literature}

Needless to say, strategic transmission of private information deals to many areas related to economics and political sciences, as clearly stated in Sobel's (2010) literature review. A quick look at the state of the art on unmediated communication classifies this type of research into two categories. First, cheap talk games, where information is unverifiable and players can lie at no cost. ${ }^{4}$ Second, games of persuasion or verifiable disclosure, since it is assumed that information is verifiable and agents can conceal information but not lie. ${ }^{5}$ Our set up is directly related with the first category.

The large strand of cheap talk literature was initiated by Crawford and Sobel (1982) where, primary related to the theory of bargaining, an informed sender sends a possibly noisy signal based on his private information to an uninformed receiver, who then takes an action that determines the welfare of both. The authors show that when there is some, but not complete, common interest imprecise talk may be necessary and sufficient to sustain credibility. This credibility constraint is necessary for equilibrium communication. Under milder conditions on that of Grawford and Sobel, the recent work by Agastya et al. (2015) completes the analysis by establishing

\footnotetext{
${ }^{4}$ See Farrell and Rabin (1996) for an exhaustive survey.

${ }^{5}$ Grossman (1981) and Milgrom (1981) are seminal papers of this line of research.
} 
that almost full revelation obtains as the two players preferences get arbitrarily close to each other.

Our main reference, Gossner et al. (2006), is also central in this line of research. In their model, a sender transmits information to the receiver without incentives to cheat. Furthermore, randomness is modeled as a binary, uniform random variable that represents the state of the world. Uncertainty is privately unveiled to the sender but not to the receiver. Although within a different modelisation of uncertainty, Agastya et al. (2014) analyze a context in which the sender has expertise on some but not all the payoff-relevant factors. Such an uncertainty can either improve or worsen the quality of transmitted information, which depends on the effective bias. For symmetrically distributed uncertainty or quadratic loss functions, the authors highlight three results: the quality of information transmission is independent of the riskiness of that uncertainty, it may be suboptimal to allocate authority to the informed player, and despite players' preferences being arbitrarily close, it is impossible to hold that the receiver prefers delegation over authority or vice versa.

Common to Crawford and Sobel, in Gossner et al.'s model, information transmission does not have an explicit cost. However, there is an implicit cost that comes from the trade-off between the cost and benefit of information transmission. Specific contexts with costly communication are offered by Sobel (2012) and Hertel and Smith (2013). In the first, it is studied the case in which both sender and receiver undertake a costly acquisition of communication capacity. The author points out that models where communication is costly and preferences are aligned can have parallel results to models of costless communication and not aligned preferences. In particular, for any communication cost or difference in preferences, full communication is not possible and failure to communicate is always possible. In the second paper, Hertel and Smith introduce discrete and costly communication in the Crawford ant Sobel's setup. The underlying idea is that words are scarce and costly. The sender can communicate only through the use of discrete messages which are ordered by cost. The state space is richer than the space of messages, since the state space is infinite while the number of messages is finite. The model captures realism since it is impossible to communicate to others the complexity of the real world. Therefore, the precision of communication may be enhanced by expending more in costly effort. In addition, the size of language endogenously emerges due to the cost of communication. As a main result, when players preferences are not aligned, an increase in communication costs may improve communication itself.

Some of the theoretical model just mentioned have been also tested in the lab. Crawford (1998) reviews the experimental literature on communication games, and 
Devetag and Ortmann (2007) critically revise some research on coordination failure in the lab experimental studies on coordination games with Pareto-ranked equilibrium. Charness and Grosskopf (2004) analyze which components might make cheap talk effective in the setting of coordination games. In particular, they design an experiment based on a two-player game to test whether information provision about the other player's action, and whether costless one-way messages before actions are taken have some influence on coordination. They find that information provision about the other person's play only enhances coordination when messages are allowed.

Through an experimental approach, Blume and Ortmann (2007) investigate the effects of costless pre-play communication in symmetric coordination games of the stag hung variety. They find that with repeated interaction cheap talk preceding games with Pareto-ranked equilibria can substantially facilitate player's coordination on the Pareto-dominant equilibrium.

The Hertel and Smith (2013) model on costly and discrete communication is contrasted in a laboratory by Duffy et al. (2014). These authors find that the size of the language endogenously emerges as a function of the costs of communication: higher communication costs are associated with a smaller language. They find that the sender payoffs, relative to equilibrium payoffs, are decreasing in cost, whereas the receiver payoffs, relative to equilibrium payoffs, are increasing in cost. Moreover, over-communication is also found.

\section{Theoretical framework}

Our theoretical set-up is based on previous work by Gossner et al. (2003, 2006).

\subsection{The one-shot game}

Consider the following game with asymmetric information. Nature, player 1 and player 2 choose an action 0 or 1 , denoted by $x, y$ and $z$ respectively.

If all agents take the same action, player 1 and 2 receive a 1 payoff, and a zero payoff otherwise. The payoff function for players in the one-shot version of this game is represented in normal form as,

$$
g(x, y, z)= \begin{cases}1 & \text { if } \quad x=y=z \\ 0 & \text { otherwise }\end{cases}
$$

It can also be represented in matrix way: 


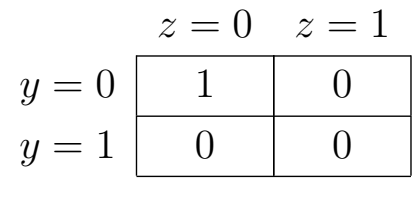

$x=0$

\begin{tabular}{l|c|c|}
\multicolumn{1}{c}{} & \multicolumn{1}{c}{$z=0$} & $z=1$ \\
\cline { 2 - 3 }$y=0$ & 0 & 0 \\
\cline { 2 - 3 }$y=1$ & 0 & 1 \\
\cline { 2 - 3 } & &
\end{tabular}

$$
x=1
$$

where nature chooses the matrix, player 1 chooses the row, and player 2 chooses the column. Players 1 and 2 have a common payoff function and, therefore, their incentives are aligned.

In the $n$-stage version of the game, nature plays a random sequence of actions denoted as $X \in\{0,1\}^{\ltimes}$ and defined as an i.i.d $\left(\frac{1}{2}, \frac{1}{2}\right)$ sequence. Before the game is played, player 1 learns the future realizations of nature, while player 2 knows only the law of the Nature's random process. Both players learn the whole history of actions pair. Formally, the strategies of the above 3-players game for players 1 and 2 are defined as,

- a (pure) strategy $Y \in\{0,1\}^{\ltimes}$ for player 1 is a sequence of mappings $Y_{t}$ : $\{0,1\}^{\ltimes} \times\{0,1\}^{t-1} \times\{0,1\}^{t-1} \rightarrow\{0,1\}$. $Y_{t}$ describes player 1's action at stage $t$, which depends on nature's sequence $X$ and players' actions in stages previous to $t$.

- a (pure) strategy $Z \in\{0,1\}^{\mathbb{N}}$ for player 2 is a sequence of mappings $Z_{t}$ : $\{0,1\}^{t-1} \times\{0,1\}^{t-1} \times\{0,1\}^{t-1} \rightarrow\{0,1\}$. $Z_{t}$ describes player 2's action at stage $t$ which depends on all past actions.

Therefore, given a sequence $X \in\{0,1\}^{\ltimes}$ for nature and a pair of strategies $(Y, Z)$ for players 1 and 2 the induced sequences of actions $\left(y_{n}\right)_{n}$ and $\left(z_{n}\right)_{n}$ of players 1 and 2 , respectively, are given by the following relations: $\left(y_{n}\right)_{n}=Y(X),\left(z_{n}\right)_{n}=Z(X, Y)$. Ultimately, player 1's actions only depend on nature's actions, while actions of player 2 depend on player 1's and nature's actions.

\subsection{Finite repetition}

In situations under asymmetric information, players may share information in order to reduce inefficiencies. Gossner et al. (2006) analyze such situations for infinite repetition. However, many real situations last a finite amount of time. We apply the techniques introduced in that paper for finitely repeated environments with asymmetric information. Let us introduce Gossner et al. strategies construction:

Players' strategies are defined over blocks of length $m<n$ in such a way that, for any nature's sequence $X=\left(x_{m}\right)_{m}$, the proportion of stages for which player 2's action 
matches nature, $z_{t}=x_{t}$, is denoted by $q \in[0,1]$. And, the proportion of stages for which player 1's action matches nature, $y_{t}=x_{t}$, conditional on $z_{t}=x_{t}$, is $p \in[0,1]$. Therefore, the proportion of stages in which $y_{t}=z_{t}=x_{t}$ is equal to $p \cdot q$. Given that strategies in this game are defined over the space $\{0,1\}, p \cdot q$ defines, in turn, each player's stage average payoff in the long-run.

Player 1 chooses a sequence $\left(y_{m}\right)_{m}$ of actions as a signal for player 2 such that:

- the number of times in which $y_{t}=z_{t}=x_{t}$ is equal to $\lfloor p \cdot q \cdot m\rfloor$.

- among the $\lceil(1-q) \cdot m\rceil$ stages in which $z_{t} \neq x_{t}$, it occurs that $y_{t}=x_{t}$ about half of the times, i.e. $\left\lfloor\frac{1-q}{2} m\right\rfloor$.

After the first block has been played, along each block player 2 has to interpret the signal sent by player 1 during the previous block and then choose her own sequence $\left(z_{m}\right)_{m}$.

Given a strategy $(p, q)$, the number of blocks of length $m$ fulfilling the above properties is computed as the product of three combinatorial numbers ${ }^{6}$. For feasibility, that result should be greater than $2^{m}$ :

$$
\left(\begin{array}{c}
m \\
m q
\end{array}\right)\left(\begin{array}{c}
m q \\
m q p
\end{array}\right)\left(\begin{array}{c}
m(1-q) \\
m(1-q) \frac{1}{2}
\end{array}\right) \geq 2^{m}
$$

\subsubsection{The information constraint}

Reescribirlo todo siguiendo OMP

The entropy function ${ }^{7}$ is used to measure the amount of information conveyed in a message transmitted through a channel. To our purpose, the entropy allows us to

${ }^{6}$ In general, $\left(\begin{array}{c}m \\ t x\end{array}\right)$ is defined as $\Gamma(m+1) /(\Gamma(x+1) \Gamma(m-x+1))$. Being $\Gamma(m)$ the Euler gamma function that satisfies $\Gamma(m)=\int_{0}^{\infty} t^{m-1} e^{-t} d t$. For $m(1-q)=1$, we consider $\left(\begin{array}{c}m(1-q) \\ m(1-q) \frac{1}{2}\end{array}\right)=2$. That means that with one digit is possible to construct the two basic sequences: 0 and 1 . 7

\subsubsection{Entropy and conditional entropy}

Let $X$ be a random variable over a finite set $\Theta$ with distribution $p$. The entropy $H(X)$ of $X$ is

$$
H(X)=-\Sigma_{\theta \in \Theta} p(\theta) \log p(\theta)=-\mathbf{E}_{X} \log p(X)
$$

where $0 \log 0=0$ (by convention $\log$ is taken in basis 2 ). The entropy of a random variable depends on its distribution only. Thus, for $p \in \Delta(\Theta)$ we let $H(p)=H(X)$ for a random variable $X$ with distribution $p$. By convention, if $p \in[0,1], H(p)$ also represents the entropy of a Bernoulli random variable of parameter $p$. 
measure the information available to players, individually or as a team. Moreover, the entropy is also useful to approximate a combinatorial number ${ }^{8}$ that connects the number of sequences given by (2) with the amount of information shared by players:

$$
2^{m H(q)} 2^{m q H(p)} 2^{m(1-q) H(1 / 2)} \geq 2^{m}
$$

where $H(q)$ is the amount of information available to player 2 and $H(p)$ the information available to player 1 . In his own interest, player 1 shares information with player 2 , gathering the total amount of information given by the joint entropy $H(p, q)$ which is defined as,

$$
H(p, q)=H(q)+q H(p)+(1-q) H(1 / 2)=H(q)+H(p \mid q) \geq 1
$$

The term $H(p \mid q)$ is the conditional entropy. The first part represents the amount of information of player 1 when his action matches nature knowing that player 2's action also does. The second part represents the amount of information of player 1 when his action matches nature knowing that player 2 does not. Player 2 is a partial informed agent with imprecise information on nature's future state, measured by $H(q)$ and less than the entropy of nature, $H(1 / 2)=1$. Therefore, the amount of information that player 1 needs to transmit to player 2 to fill in the gap of information is $1-H(q) \leq H(p \mid q)$. Player 1 does not need to transmit all his private information to improve his own earnings, and sharing just a part of it is enough for both players to have incentives to design a joint block strategy $(p, q)$ that will match nature $p q m$

Given a pair of random variables $\left(X_{1}, X_{2}\right)$ taking values in $\Theta_{1} \times \Theta_{2}$ with joint distribution $p\left(\theta_{1}, \theta_{2}\right)$, we denote by $p\left(\theta_{2} \mid \theta_{1}\right)$ the conditional probability that $X_{2}=\theta_{2}$ given that $X_{1}=\theta_{1}$. Define $h\left(X_{2} \mid \theta_{1}\right)=-\Sigma_{\theta_{2} \in \Theta_{2}} p\left(\theta_{2} \mid \theta_{1}\right) \log p\left(\theta_{2} \mid \theta_{1}\right)$. Thus $h\left(X_{2} \mid \theta_{1}\right)$ is the entropy of $X_{2}$ when the realization $X_{1}=\theta_{1}$ is known.

The conditional entropy $H\left(X_{2} \mid X_{1}\right)$ of $X_{2}$ given $X_{1}$ is

$$
H\left(X_{2} \mid X_{1}\right)=\mathbf{E}_{X_{1}}\left[h\left(X_{2} \mid X_{1}\right)\right]=\sum_{\theta_{1} \in \Theta_{1}} p\left(\theta_{1}\right) h\left(X_{2} \mid \theta_{1}\right)
$$

Direct computation shows that $H\left(X_{1}, X_{2}\right)=H\left(X_{1}\right)+H\left(X_{2} \mid X_{1}\right)$. This extends to a family of random variables $\left(X_{1}, \ldots, X_{n}\right)$ to:

$$
H\left(X_{1}, \ldots, X_{n}\right)=H\left(X_{1}\right)+\sum_{k=2}^{n} H\left(X_{k} \mid X_{1}, \ldots, X_{k-1}\right)
$$

The entropy of a number $0<x<1$ is defined as $H(x)=-x \log _{2} x-(1-x) \log _{2}(1-x)$. The entropy is minimal for $x=\{0,1\}, H(0)=H(1)=0$, and maximal for $x=1 / 2, H(1 / 2)=1$.

${ }^{8}$ For $0<x<1$, the combinatorial number $\left(\begin{array}{c}m \\ m x\end{array}\right)$ is upper bounded by $2^{m H(x)}$. 
times within a block. The remaining $(1-p q) m$ times are errors used to transmit information about the next block.

The fact that the informa- tion used by the agent cannot exceed the information received leads to an information-theoretic inequality expressed using the Shannon [13] entropy function, and which we call the information constraint.

Since the coded information is embedded into the error locations, there is a tradeoff between earnings and errors: the fewer the errors, the more coordination and the higher earnings for players, but the fewer chances to inform on nature's future state, which in turn reduces future earnings.

Ultimately, the number of errors depends on the strategy $(p, q)$ that, for feasibility, must satisfy the information constraint given in (3). Players choose a joint strategy from the set of feasible strategies $S$. Let $S$ be the set of pairs $(p, q)$ verifying (3). Taking logarithms, set $S$ is defined as,

$$
S=\{(p, q): H(q)+q H(p)+(1-q) \geq 1\}
$$

Notice that if $p=1$ and $q=1$, both players have perfect information and the information constraint does not work: $H(1)+1 H(1)+(1-1)=0<1$. Consequently, we have that the set $S$ is a proper subset of $[0,1] \times[0,1]$. Moreover, the information constraint does not depend on the length of the block $m$.

\subsubsection{Coordination strategies in $\mathbb{Q}$}

In order to coordinate actions and maximize payoffs, players need to transmit information through actions. In a way, players need to perform a communication system on finite sequences. To do that, the number of matches must be defined as an integer number and additional definitions are needed into the rational number set $\mathbb{Q}$.

Given a strategy $(p, q)$ on $S$, let us define the counterpart in rational numbers as $\tilde{q}(m)=\frac{\lfloor q m\rfloor}{m}, \tilde{p}(m)=\frac{\lfloor p q m\rfloor}{\lfloor q m\rfloor}, m$ is the size of the block. If $q \in[0,1]$ then $\tilde{q} \in$ $\left\{0, \frac{1}{m}, \ldots, \frac{m-1}{m}, 1\right\}$. Similarly, since $p \in[0,1]$ then $\tilde{p} \in\left\{0, \frac{1}{m \tilde{q}}, \ldots, \frac{m \tilde{q}-1}{m \tilde{q}}, 1\right\}$.

The expression (2) can now be rewritten with rational numbers as,

$$
\left(\begin{array}{c}
m \\
m \tilde{q}
\end{array}\right)\left(\begin{array}{c}
m \tilde{q} \\
m \tilde{q} \tilde{p}
\end{array}\right)\left(\begin{array}{c}
m(1-\tilde{q}) \\
m(1-\tilde{q}) \frac{1}{2}
\end{array}\right) \geq 2^{m}
$$

also the information constraint (4) as,

$$
H(\tilde{q}(m))+\tilde{q}(m) H(\tilde{p}(m))+(1-\tilde{q}(m)) \geq 1
$$


and we will refer to this constraint as the rational information constraint.

Denote by $\tilde{S}_{m}$ the set of pairs $(\tilde{p}, \tilde{q})$ in rational number verifying the rational information constraint ${ }^{9}$ :

$$
\tilde{S}_{m}=\left\{(\tilde{p}, \tilde{q}) \in \frac{\mathbb{Z}}{m \mathbb{Z}} \times \frac{\mathbb{Z}}{m \mathbb{Z}}: \begin{array}{r} 
\\
\tilde{q}(m)=\frac{\lfloor q m\rfloor}{m}, q \in[0,1] \\
\tilde{p}(m)=\frac{\lfloor p q m\rfloor}{\lfloor q m\rfloor}, p \in[0,1] \\
H(\tilde{q})+\tilde{q} H(\tilde{p})+(1-\tilde{q}) \geq 1\}
\end{array}\right.
$$

Remark 1 The rational information constraint depends on the size of the block $m$.

$$
H(\tilde{q}(m))+\tilde{q}(m) H(\tilde{p}(m))+(1-\tilde{q}(m)) \geq 1
$$

The following lemma states the existence of rational joint strategies for players 1 and 2 given a fixed length for the block.

Lemma 2 Let $n>0$

- There exists $m \mid n$ such that $\tilde{S}_{m} \neq 0$.

- Let $D_{n}=\left\{m \mid n: \tilde{S}_{m} \neq 0\right\}$. There exists $m^{*} \in D_{n}$ and $\left(p^{*}, q^{*}\right) \in \tilde{S}_{n^{*}}$ such that $p^{*} q^{*}$ is maximal over $(\tilde{p}(m), \tilde{q}(m)) \in \tilde{S}_{m}, \forall n \in D_{n}$.

\section{Proof.}

- For all $m \in D_{m}$ consider the family $\left\{\frac{\lfloor q m\rfloor}{n}, \frac{\lfloor p q m\rfloor}{\lfloor q m\rfloor}\right\}_{m \in D_{n}}$.

Observe that $\tilde{S}_{m} \subset[0,1] \times[0,1]$ is a compact set. Therefore, the product $\tilde{p}(m) \tilde{q}(m)$ reaches its maximal value in this set.

From the set of pairs $(\tilde{p}(m), \tilde{q}(m))$ that verify the rational information constraint:

$$
H(\tilde{q}(m))+\tilde{q}(m) H(\tilde{p}(m))+(1-\tilde{q}(m)) \geq 1
$$

We obtain the optimal pair $\left(p^{*}, q^{*}\right)$ such that the product $p^{*} q^{*}$ reach the maximal value in $\tilde{S}_{m}$.

\footnotetext{
${ }^{9} \mathbb{Z}$ denotes the set of positive integer numbers.
} 
As the entropy approximation provides an upper bound for a combinatorial number, the rational information constraint (10) is a necessary but not sufficient condition for a strategy $(\tilde{p}(m), \tilde{q}(m))$ to be implementable. Specifically, in the case of blocks of short length like $m=\{3,4\}$, a strategy may verify the rational information constraint but not the combinatorial inequality (6). To overcome such cases, we introduce a refinement for a strategy to be implementable and define the implementable information constraint in combinatorial terms as,

$$
\left(\begin{array}{c}
m \\
m \tilde{q}
\end{array}\right)\left(\begin{array}{c}
m \tilde{q} \\
m \tilde{q} \tilde{p}
\end{array}\right)\left(\begin{array}{c}
m(1-\tilde{q}) \\
m(1-\tilde{q}) \frac{1}{2}
\end{array}\right)+\left(\begin{array}{c}
m \\
m
\end{array}\right)\left(\begin{array}{c}
m \\
m(1-\tilde{q} \tilde{p})
\end{array}\right) \geq 2^{m}
$$

Notice that we have added a second term to the left-hand side of inequality (6), representing the total number of $m$-length sequences with a number of signaling errors equals $m(1-\tilde{q} \tilde{p})$.

\section{Experimental set-up}

In this subsection we determine and justify the criteria that a sequence and block lengths has to fulfill in order to be adequate for its implementation in the lab. ${ }^{10} \mathrm{We}$ then define the optimal strategies for the chosen length of the sequence. Finally, we describe in detail the design of the experimental session undertaken.

\subsection{Sequence and block lengths}

In order to find the "right" length of the sequence to be used in the experiment, we explore several sequence lengths $(n)$ combined with several block lengths $(m)$. In fact we look for the block strategy $(\tilde{p}(m), \tilde{q}(m))$ of length $m<n$ that maximizes earnings in the length of the sequence $(n)$.

Let us denote as $G(n, m)$ the total payoff obtained in a sequence of length $n$ by a block strategy of length $m: G(n, m)=\tilde{p}(m) \tilde{q}(m) m_{b}(n, m) m$. Being $m_{b}(n, m)$ the number of blocks of length $m$ in the sequence of length $n$ as follows:

$$
m_{b}(n, m)= \begin{cases}\left\lfloor\frac{n}{m}\right\rfloor & \text { if } n \text { modulo } m \geq m(1-\tilde{p}(m) \tilde{q}(m)) \\ \left\lfloor\frac{n}{m}\right\rfloor-1 & \text { otherwise }\end{cases}
$$

\footnotetext{
${ }^{10} \mathrm{~A}$ more extended and preliminary version of this analysis is included in García-Gallego et al. (2015).
} 
Because in the first block players are not able to transmit any information but just play, any matching in that block occurs by chance. Let $n$ modulo $m$ be the remainder of the fraction between $n$ and $m$. If $n$ modulo $m$ is greater than or equal to the number of errors that are necessary to communicate, i.e $m(1-\tilde{p}(m) \tilde{q}(m))$, then the number of blocks generating payoff is given as the minimum integer number $\left\lfloor\frac{n}{m}\right\rfloor$. Otherwise, the number of blocks is $\left\lfloor\frac{n}{m}\right\rfloor-1$.

Given $m$, the optimal total earning $G^{*}(n, m)$ can be obtained by a block strategy that maximizes the total earnings: $G^{*}(n, m)=\max _{m} G(n, m)$. Since the solution to this problem is not unique for $n$, it is established as the optimal block strategy $\left(\tilde{p}\left(m^{*}\right), \tilde{q}\left(m^{*}\right)\right)$ the one that has the minimum length, i.e. $m^{*}=\min _{m} \arg \left(G^{*}(n, m)\right)$

We consider that lengths, in order to be implementable in the lab, should be long enough for subjects to learn during the game and should have an optimal strategy easy to be designed during the pre-play stage of the game.

We develop an algorithm that solves the min-max problem. In the first place, we apply lemma 2 in order to construct strategy sets $\tilde{S}_{m}^{*}$. Divide the interval $[0,1]$ into $m$ disjoint intervals such that:

$$
\frac{x}{m} \leq \tilde{q}(m)<\frac{x+1}{m}, \quad x=0,1, \ldots, m
$$

and call $x$ the number of times player 2 matches nature. For each one of $m$ intervals a rational number exists in the set:

$$
\tilde{q}(x \mid m)=\left\{0, \frac{1}{m}, \frac{2}{m}, \ldots, \frac{m-1}{m}, 1\right\}
$$

Let $\tilde{p}(m)$ be conditional on $\tilde{q}(m)$ such that:

$$
\frac{y}{x} \leq \tilde{p}(m)<\frac{y+1}{x}, \quad y=0,1, \ldots, x, \quad x>0
$$

For each one of $m$ intervals a set exists expressed as:

$$
\tilde{p}(y \mid x, m)=\left\{\frac{0}{x}, \frac{1}{x}, \ldots, \frac{x-1}{x}, 1\right\}, x \neq 0
$$

By programming with Mathematica 7.0 , we provide strategy sets $\tilde{S}_{m}$. Table 4 in the Appendix shows the set of optimal strategies verifying the rational information constraint. As an example, $m=2,3,4,5$ the sets are:

$$
\begin{aligned}
& \tilde{S}_{2}=\left\{\left(\frac{1}{2}, 1\right),\left(1, \frac{1}{2}\right)\right\} \\
& \tilde{S}_{3}=\left\{\left(1, \frac{1}{3}\right),\left(\frac{1}{2}, \frac{2}{3}\right),\left(1, \frac{2}{3}\right)\right\}
\end{aligned}
$$




$$
\begin{aligned}
& \tilde{S}_{4}=\left\{\left(\frac{1}{2}, 1\right),\left(1, \frac{1}{4}\right),\left(\frac{1}{2}, \frac{1}{2}\right),\left(1, \frac{1}{2}\right),\left(\frac{1}{3}, \frac{3}{4}\right),\left(\frac{2}{3}, \frac{3}{4}\right),\left(1, \frac{3}{4}\right)\right\} \\
& \tilde{S}_{5}=\left\{\left(1, \frac{1}{5}\right),\left(\frac{1}{2}, \frac{2}{5}\right),\left(1, \frac{2}{5}\right),\left(\frac{1}{3}, \frac{3}{5}\right),\left(\frac{2}{3}, \frac{3}{5}\right),\left(1, \frac{3}{5}\right)\left(\frac{1}{4}, \frac{4}{5}\right),\left(\frac{1}{2}, \frac{4}{5}\right),\left(\frac{3}{4}, \frac{4}{5}\right)\right\}
\end{aligned}
$$

Observe, for example, that the strategy $\left(1, \frac{3}{4}\right)$ in $\tilde{S}_{4}$ does not fulfill the implementable information constraint given in (11):

$$
\left(\begin{array}{l}
4 \\
3
\end{array}\right)\left(\begin{array}{l}
3 \\
3
\end{array}\right)\left(\begin{array}{c}
1 \\
1 / 2
\end{array}\right)+\left(\begin{array}{l}
4 \\
4
\end{array}\right)\left(\begin{array}{l}
4 \\
1
\end{array}\right)=12 \not 2^{4}=16
$$

That means that it is not possible to construct blocks of length $m=4$ with only one signaling mistake. Furthermore, note that 2-length blocks and 3-length blocks are implementable strategies that need only one signaling mistake.

Given a set of strategies, we are able to optimal strategies by maximizing total earnings per block. For the sets just considered above, the optimal strategies are:

$$
\begin{aligned}
& \tilde{S}_{2}^{*}=\left\{\left(\frac{1}{2}, 1\right),\left(1, \frac{1}{2}\right)\right\} \\
& \tilde{S}_{3}^{*}=\left\{\left(1, \frac{2}{3}\right)\right\} \\
& \tilde{S}_{4}^{*}=\left\{\left(\frac{2}{3}, \frac{3}{4}\right)\right\} \\
& \tilde{S}_{5}^{*}=\left\{\left(1, \frac{3}{5}\right),\left(\frac{3}{4}, \frac{4}{5}\right)\right\}
\end{aligned}
$$

Once the optimal strategies are identified, the next step is evaluating each strategy calculating the earnings associated with them for a given sequence length $n=$ $5,6, \cdots, N$. Each combination $(n, m)$ leads to $G(n, m)$ total earnings.

In addition, given that this problem has multiple solutions for the optimal block length $m^{*}$, we require that, for a given sequence length $n$, the length of the block to be minimal, that is, $m^{*}=\min _{m} \arg \left(G^{*}(n, m)\right)$.

Table 7 in the Appendix reports the optimal implementable strategies. Taking into account all the requirements for a length to be reasonably implementable in the lab, we choose $n=55$. To this sequence length optimally corresponds the block length $m=3$ and the strategy $\left(1, \frac{2}{3}\right)$ known as majority rule for 3-length blocks, that we explain next. 


\subsection{The majority rule for 3-length block strategy}

The 3-length block strategy ${ }^{11}$ strategy is ruled such that in each block after the first stage, player 2's actions match nature's actions in at least 2 out of the 3 stages. Player 2 's triple action is either $(0,0,0)$ or $(1,1,1)$ in each block, whereas player 1's actions signal to player 2 the majority action of nature in the next block. This signaling is achieved by playing nature's majority action of the next block in a signaled stage of the current block. If actions of player 2 match nature's at all stages of the current block, then the third stage of the block is the one signaled to signal the majority rule for the next block. If the actions of player 2 match the sequence of nature in exactly two out of three stages, the mismatched stage is the one signaled. That strategy guarantees a per stage payoff of $2 / 3$.

Table 1 shows the 3-length majority rule strategy. Note that there is only one mistake signaled by player 1's actions (in bold) and that the total guaranteed payoff is at least $10(2 \times 5$ blocks $)$, and the guaranteed stage average payoff is $\frac{10}{16}=0.625$. Furthermore, the signaling action may match the majority action of the current block $\frac{1}{4}$ of the times. Thus, there is an additional expected payoff equal to $1.25\left(\frac{1}{4} \times 5\right.$ blocks $)$. This implies that the stage expected payoff is $\frac{11.25}{16}=0.70$.

Table 1: Majority rule strategy

\begin{tabular}{|c|c|ccc|ccc|ccc|ccc|ccc|}
\hline \hline Slayers & 1 & 2 & 3 & 4 & 5 & 6 & 7 & 8 & 9 & 10 & 11 & 12 & 13 & 14 & 15 & 16 \\
\hline Nature & 1 & 1 & 0 & 0 & 1 & 0 & 1 & 1 & 1 & 1 & 0 & 1 & 0 & 0 & 0 & 0 \\
\hline 1 & $\mathbf{0}$ & $\mathbf{1}$ & 0 & 0 & 1 & $\mathbf{1}$ & 1 & 1 & 1 & $\mathbf{0}$ & 0 & $\mathbf{0}$ & 0 & 0 & 0 & 0 \\
\hline 2 & $*$ & 0 & 0 & 0 & 1 & 1 & 1 & 1 & 1 & 1 & 0 & 0 & 0 & 0 & 0 & 0 \\
\hline Payoff & $*$ & $\mathbf{0}$ & 1 & 1 & 1 & $\mathbf{0}$ & 1 & 1 & 1 & $\mathbf{0}$ & 1 & $\mathbf{0}$ & 1 & 1 & 1 & 1 \\
\hline \hline
\end{tabular}

\footnotetext{
${ }^{11}$ The possible binary sequences of length 3 are: $(0,0,0),(0,0,1),(0,1,0),(1,0,0),(1,1,0),(1,0,1)$, $(0,1,1)$ and $(1,1,1)$. There are four sequences with majority rule 0 , and also four with majority rule 1. The probability of the majority rule 'equals 0 ' is given by $\operatorname{prob}($ majority $=0)=\operatorname{prob}(000 \cup$ $001 \cup 010 \cup 100)=4 \frac{1}{8}=\frac{1}{2}$. Similarly, the probability of the majority rule 'equals 1 'is equal to $\operatorname{prob}($ majority $=1)=\operatorname{prob}(110 \cup 101 \cup 011 \cup 111)=4 \frac{1}{8}=\frac{1}{2}$. Thus, the probability of two consecutive blocks have the same majority is $\frac{1}{2}$. The probability that an intended mistake (say $x)$ becomes a random match is equal to: $P(x=$ majority $=0) P($ majority $=0) P($ majority $=$ $0)+P(x=$ majority $=1) P($ majority $=1) P($ majority $=1)=\frac{1}{4}$.
} 


\subsection{Experimental design}

The experiment was run at the experimental economics lab at the University of Valencia (LINEEX) in Spain, and consisted of two sessions of 60 subjects each, all third and fourth year students of Economics, International Business and Business Administration at that University. In each session, students were grouped in pairs and randomly assigned a permanent role: Type 1 (plays player 1 ) or Type 2 (plays player 2). ${ }^{12}$

At the beginning of the session and before the matching, students performed several tests. First, the Cognitive Reflexion Test $(\mathrm{CRT})^{13}$ Second, a Team Work Test (TWT) of twenty five questions. We used subjects' performance in the TWT to rank students from more to less collaborative. Thirty pairs were formed by taking consecutive people two by two. This way, the pair number 1 was composed by the two most collaborative ones, and the pair number 30 was formed by the two less collaborative ones in the sample.

After doing the matching, the instructions of the experiment were given to subjects. ${ }^{14}$ The decision making of subjects in this experiment consist in playing the matching pennies repeated game introduced in section 3: a no-player, called Prize, was defined as an i.i.d random variable taking value 0 or 1 with probability $\left(\frac{1}{2}\right)$. A 55-length random sequence was generated at the beginning of the play phase by a random number generator. This was common knowledge to both players. Immediately after, the Type 1 player knew the complete sequence of Prize, while the player Type 2 did not. After that, subjects started playing the game and, at the beginning of a new round, each player knew the actions played in the past. Players payoffs were defined such that, in each round, both players get 1 only if both of them match the action played by Prize, and zero otherwise. No losses are possible.

In an experimental session, players play twice the coordination game. Specifically, a session was divided in two parts: In the first part, named Play 1, subjects played the coordination game during 55 rounds. Immediately after, the second part, named Play 2, is played. Play 2 is exactly the same as Play 1 but nature plays a different sequence. Both parts in a session have the same structure: first, the 55 sequence of nature's actions is generated and privately transmitted to Type 1 player, and then both players play the repeated game. At the end of each round, subjects are privately informed about all actions taken and about individual earnings in that round. As a result, the two parts of a session differ just in the fact that, when Play 2 starts,

\footnotetext{
${ }^{12}$ This experiment is part of the more extended experimental study in García-Gallego et al. (2013).

${ }^{13}$ This test measures how reflective a person is in his decision making.

${ }^{14}$ See the translated version from the original in Spanish in the Appendix.
} 
subjects have already played Play 1 . At the end of each Play, subjects were privately informed about their final earnings in that part.

Each part, Play 1 and Play 2, had also a pre-play stage of 3 minutes-chat before the coordination game is played. During the time of the chat, subjects were allowed to send free-form messages to each other to share information and experience. Subjects could finish the chat at any moment. Otherwise, the chat would automatically close once after the three minutes. Once the chat was finished, Type 1 was informed in private about the sequence of Player 1 and the game started.

The two sequences of a session were randomly generated at the beginning of each part of the session through a random number generator simulating a ' 0 ' and ' 1 ' binary variable, each outcome with a constant probability of $1 / 2$. Subjects were informed about the computerized random process as being like tossing a coin.

A pilot session of 8 periods was ran for subjects to have an accurate understanding of the frame of the experiment. Once the pilot finished, the real experiment started. ${ }^{15}$

At the end of the session, each participant was privately paid in cash. Particularly, as specified in the instructions, a subject was paid 1 ECU (Experimental Currency Unit) per round in which all strategies matched. The ECU/Euro exchange rate was $1 \mathrm{ECU}=\frac{1}{4}$ Euro. Average payoffs per subject were around 18 Euros.

\section{Data analysis and main results}

In this section, we first conduct a statistical analysis on the entire sample of the number of matchings as well as on the respective subsamples corresponding to the Play 1 and Play 2, second conduct a cluster analysis to identify coordination levels, then analyze CRT and TWT results including a correlation analysis between CRT and TWT scores and the number of matchings, and finally perform an econometric estimation of the theoretical model.

Table ?? reports the main statistics on the number of matchings. In median, the overall number of matchings is 32 , which represents the $58 \%$ of success in the 55 rounds of a play. It emerges that number of rights significantly increases with experience: subjects improve coordination in the Play 2. In fact, according to Wilcoxon signedrank test for the equality of medians, there exists a powerful significant difference between plays $(z=3.701, p=0.0002)$.

\footnotetext{
${ }^{15}$ Data from the pilot periods are not reported in our results.
} 
Table 2: Statistics on matching by play

\begin{tabular}{|c||c|c||c|}
\hline \hline $\begin{array}{c}\text { Descriptive } \\
\text { Statistics }\end{array}$ & Play 1 & Play 2 & All \\
\hline Max. & 42 & 44 & 44 \\
Min. & 18 & 18 & 18 \\
Average & 30.08 & 33.65 & 31.87 \\
Median & 30 & 34 & 32 \\
St.D & 5.12 & 5.72 & 5.7 \\
Rate & 0.55 & 0.62 & 0.58 \\
Obs. & 60 & 60 & 120 \\
\hline
\end{tabular}

'Rate' refers to a matching percentage defined as the quotient between the median and the length of sequence $\mathrm{n}=55$.

Result 1: Experimental subjects coordinate their actions with experience, arriving to high levels of coordination in the second play of the session.

We follow to identify the characteristics of coordination clusters by executing the K-MEANS algorithm (MacQueen, 1967) on the entire sample of the number of matchings ${ }^{16}$, and then within each cluster strategies are separated by plays. As reported in table ??, we identify three clusters corresponding to three coordination levels:

- Cluster $L$ includes the poorest strategies: strategies in which player 1 plays nature's action and player 2 plays at random. Both strategies are naive and have a correspondence with the theoretical $(p, q)=\left(1, \frac{1}{2}\right)$, an expected payoff of $\frac{1}{2}$ per stage and a total expected payoff over the whole sequence of $55 \times \frac{1}{2}=27.5$. In practice, 38 out of 120 lab strategies were classified as low coordination and produced the $47 \%$ of success, in median.

- Cluster $M$ includes suboptimal strategies like the one of 2-length block, with a guaranteed per period payoff of 1 and a per period expected payoff of $\frac{5}{8}$. Therefore, the total payoff lays within the interval [27.5,34.37] for the 55-length sequence. Regarding the lab strategies, 29 out of 120 were classified as medium coordination with a rate of success of the $56 \%$.

\footnotetext{
${ }^{16} \mathrm{~K}$-means algoritm is implemented in the scientific program Matlab. The distance measure applied is the sum of absolute differences, known as the $L 1$ distance. Each centroid is the componentwise median of the points in that cluster: $d(x, c)=\sum_{j=1}^{p}\left|x_{j}-c_{j}\right|$.
} 
- Cluster $H$ includes the optimal 3-length block strategies, with a guaranteed per period payoff of $\frac{2}{3}$ and a per period expected payoff of $\frac{3}{4}$. The total payoff for the whole sequence lays in the interval $[36.66,41.25]$. The number of strategies implemented in the experiment considered as high coordination was 53 out of 120 , that means a success of $67 \%$. In spite of the surprising number such strategies, only 2 out of 53 performed the optimal coding rule.

Table 3: Statistics on matching within coordination clusters

\begin{tabular}{|c||c|c|c||c|c|c||c|c|c|}
\hline \hline \multicolumn{1}{|c||}{ Descriptive } & \multicolumn{3}{c||}{ Play 1 } & \multicolumn{3}{c||}{ Play 2 } & \multicolumn{3}{c|}{ All } \\
\cline { 2 - 10 } Statistics & $\mathrm{L}$ & $\mathrm{M}$ & $\mathrm{H}$ & $\mathrm{L}$ & $\mathrm{M}$ & $\mathrm{H}$ & $\mathrm{L}$ & $\mathrm{M}$ & $\mathrm{H}$ \\
\hline Average & 24.67 & 29.63 & 35.35 & 26.65 & 32.15 & 38.27 & 25.55 & 30.76 & 37 \\
Median & 26 & 30 & 35 & 27 & 32 & 39 & 26 & 31 & 37 \\
St.D & 2.52 & 1.78 & 2.31 & 3.55 & 2.19 & 2.41 & 3.14 & 2.32 & 2.76 \\
Rate & 0.47 & 0.55 & 0.64 & 0.49 & 0.58 & 0.71 & 0.47 & 0.56 & 0.67 \\
Obs. & 21 & 16 & 23 & 17 & 13 & 30 & 38 & 29 & 53 \\
\hline
\end{tabular}

'Rate' refers to a matching percentage defined as the quotient between the median and the length of sequence $\mathrm{n}=55$.

As mentioned in the subsection 4.3 the participants of the experiment were face a Cognitive Reflection Test (CRT) composed by 3 questions and a Team Work Test (TWT) of 25 questions $^{17}$ in the likert scale from 1 to 4 , meaning 1 no cooperative at all and 4 absolutely cooperative ${ }^{18}$. Participants, separate by role player 1 and player 2, were sorted in decreasing order according to their TWT score, and then each participant was paired to the counterpart in the same order to form a permanent pair. Thus, the pair 1, the highest scored pair, score would be presumably the most inclined to coordinate each other, whereas the pair 30, the lowest scored pair, would be the less inclined to coordinate each other. Therefore, we would expect to find a positive relation between coordination and TWT score in our experiment.

Figure 1 plots the distributions of TWT and CRT scores. Panel (a) shows the overall distributions by types of players, which indicate homogenous groups regarding to subjects' attitudes towards cooperation and reflection. Furthermore, by coordination levels, subjects exhibit no significant differences in such attitudes: median TWT(CRT) score was around 78(13.98), in overall. Panel (b) represents the distribution of players' TWT scores by play and cluster. Looking at the left of the graph,

\footnotetext{
${ }^{17}$ See appendix for question test.

${ }^{18}$ The maximal score is 100 and the minimal score is 25 .
} 
we find that 12 out of 21 pairs coordinated at level L in Play 1 enhanced coordination in Play 2. In contrast, at the right of the graph, we find that 7 out of 23 pairs coordinated at level $\mathrm{H}$ in Play 1 diminished coordination in Play 2. The distributions of TWT scores by coordination levels look like really different, which indicates that there is no relation between such scores and coordination. In fact, the coefficient of Pearson's correlation between the number of matchings and TWT scores is not significant, neither between the number of matchings and CRT scores nor between TWT scores and CRT scores. This evidence leads to the following result:

Result 2: Experimental subjects' attitudes towards reflection and cooperation do not matter to coordination. 


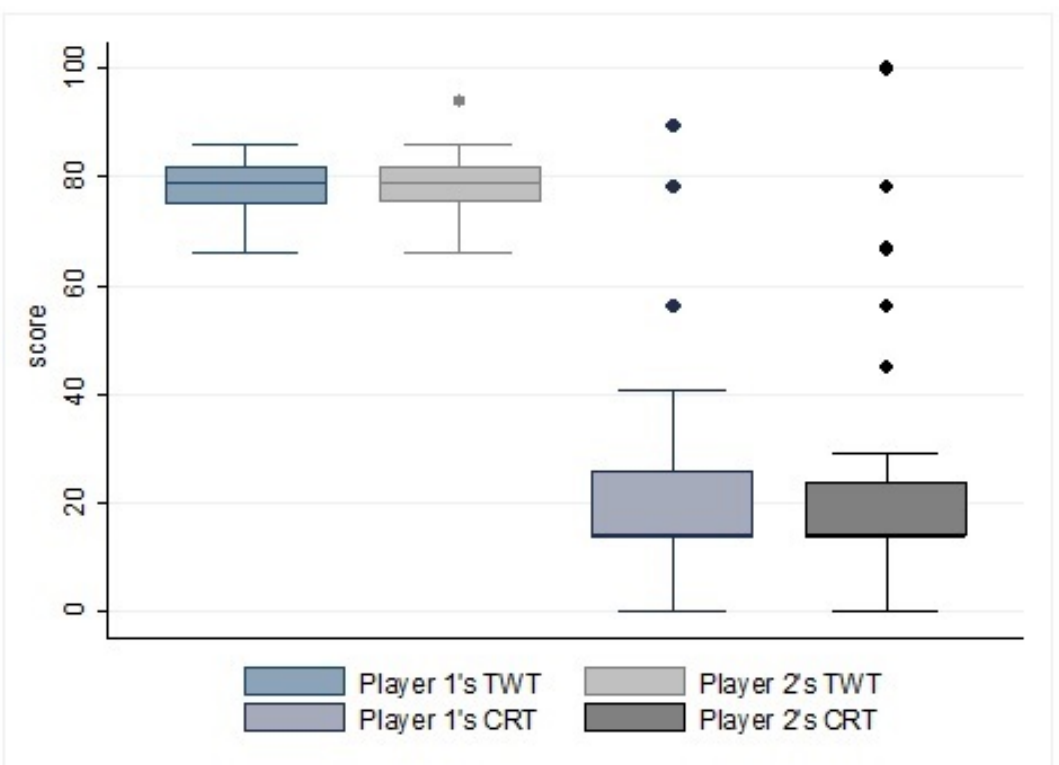

(a) TWT and CRT scores by type of player

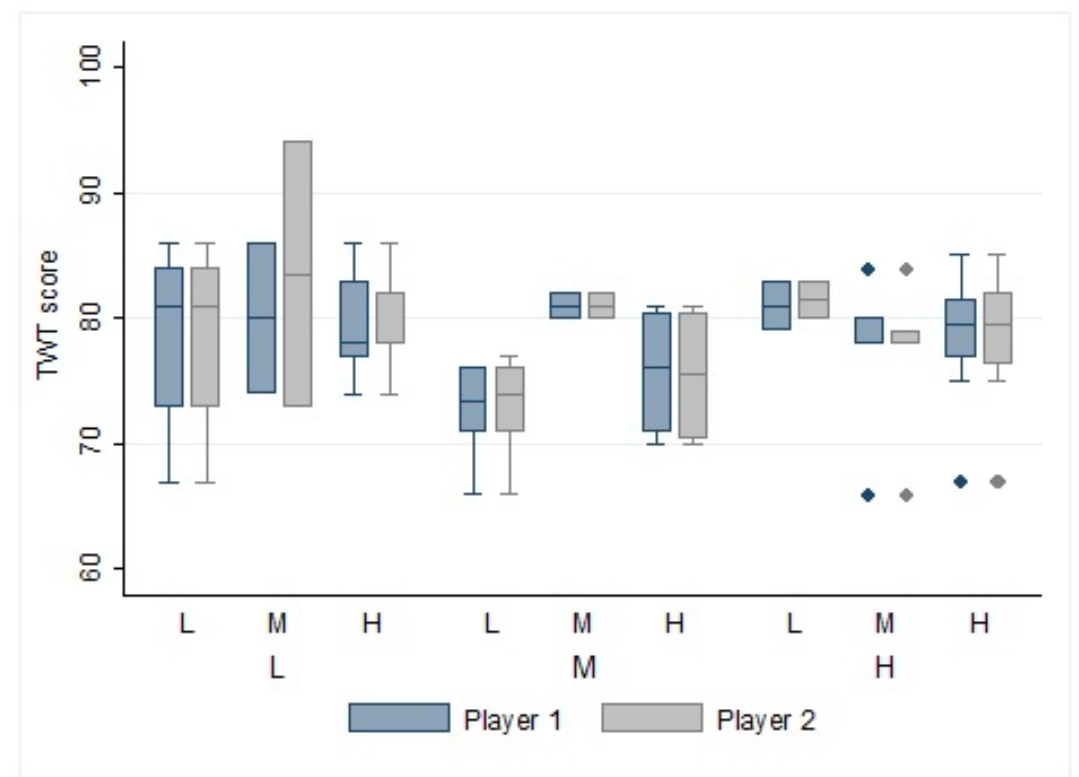

(b) TWT scores by cluster and type of player

Figure 1: Distributions of CRT and TWT scores

\subsection{Coordination strategies $(p, q)$ in the lab}

In the pure matching pennies game coordination is tacit and random. In contrast, in our matching pennies game coordination is also tacit but not random.

Players jointly define a coordination strategy before playing the game, which allows to assure their matching and payoff. Superior coordination strategies convey 
more information that player 2 decodes according to a joint coordination rule and matches nature's action in proportion q.Player 1 makes one or more mistakes to inform his partner about nature future playing. Thus, player 1 matches both player 2 and nature's actions in the proportion $p_{1}$, while matches just nature's actions in proportion $p_{2}$.

As far as the corresponding experimental strategies for proportions $(p, q)$, as already defined in Section 3, the evidence is the following:

- Figure 2 reports proportions $q$ in Plays 1 and 2 distributed by coordination cluster. Firstly, the median values are increasing in coordination, and higher in the Play 2. In overall, there exists a powerful significant difference of medians between both plays $(z=-3.610, p=0.0003)$.

- However, in the case of proportions $p_{1}$ and $p_{2}$ no differences of medians are found between plays $(z=0.936, p=0.3494 ; z=-0.738, p=0.4608) .{ }^{19}$

- Regarding the relation between player 1 's and player 2's decisions. On the one hand, the proportion $p_{1}$ exhibits an overall negative coefficient of Pearson's correlation with the proportion $q($ corr $=-0.474, p=0.0001)$. In other words, because player 1 uses mistakes to inform the player 2 about the nature's next actions, the higher $p_{1}$ is the lower the number of mistakes is and therefore the less information is transmitted and, as a result of coordination strategy, the lower the number of player 1's chances to match nature $q$ is. On the other hand, the proportion $p_{2}$ is uncorrelated with the proportions $q$ and $p_{1}$ since the corresponding coefficient of correlations are not statistically significant at conventional levels. That means that the player 1's decision is random (theoretical optimal value is $p_{2}=\frac{1}{2}$ ) when player 2 does not match nature.

All these findings lead to our third result.

Result 3: The behavior of player 1 induces the behavior of player 2. Being information transmission based on the mistakes $\left(1-p_{1}\right)$ of player 1 that are interpreted by and converted in rights $(q)$ of player 2 .

\footnotetext{
${ }^{19}$ It is applied Wilcoxon signed-rank test for the equality of medians.
} 


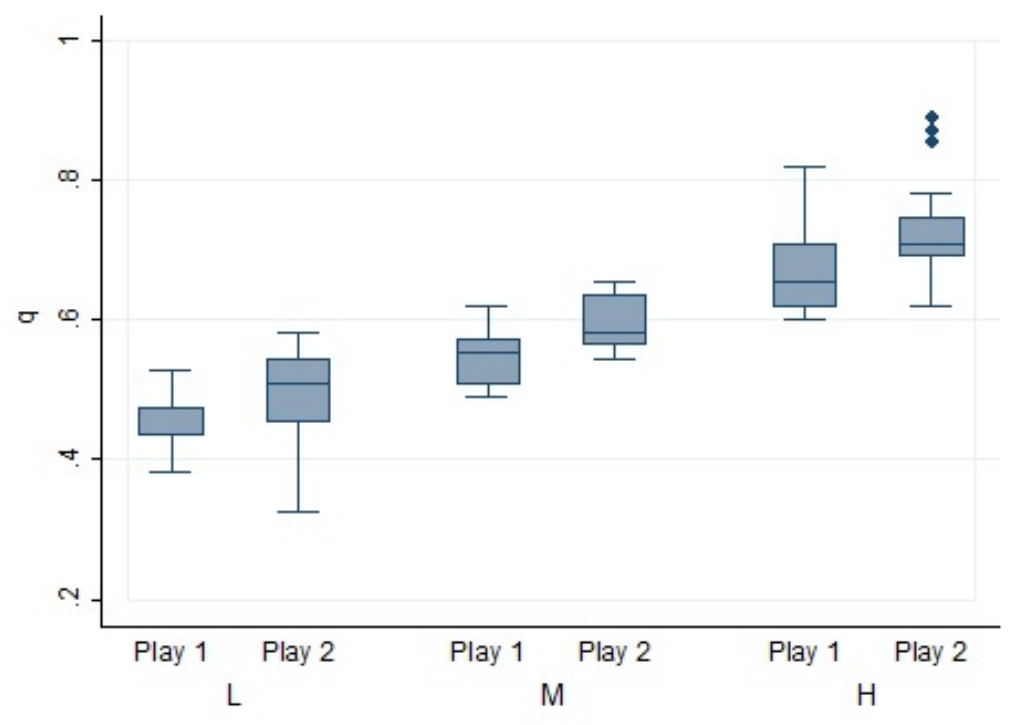

Figure 2: Player 2's matching frequency by cluster

\subsection{Logit model estimation}

According to the theoretical model, codification rules implicitly define player 1 and player 2 matching proportions $p$ and $q$, which, in turn, determine the long term average payoff $(p \cdot q \cdot n)$. In previous sections, we characterized the optimal pair $(p, q)$ $=\left(1, \frac{2}{3}\right)$ for a finite sequence of nature of length 55 , being the corresponding strategy the majority rule for 3-length blocks. In this subsection, we estimate the models of players behavior by binary logit models.

We first estimate the model of player 2. If information transmission exists, the decisions of players 2 will depend on nature and the previous decisions of players 1 , which is included by lagging one and two periods the variable representing the decisions of player 1. Also, dummies variables to catch the effect of coordination clusters $\mathrm{M}$ and $\mathrm{H}$ are added. Furthermore, the model estimation with the entire sample include dummies per session and play.

Regarding the estimation of the model of player 1 , we estimate the probability $p_{1}$, which is conditional on the player 2 matches nature's action, and then estimate the probability $p_{2}$, which is conditional on the player 2 does not match nature's action. In this case, the only explanatory variable is nature's action and also dummies per cluster and session are included.

Table 6 reports the logistic regression marginal effects of probabilities $q, p_{1}$, and $p_{2}$ for the entire sample and each play.

We find a positive effect of nature intervention on $q$. In other words, when na- 
ture's action changes from ' 0 ' to ' 1 ', then $q$ increases, and viceversa. Concerning the entire sample, we find significant both the one and two lagged actions of player 1. When player 1 changes his action from ' 1 ' to ' 0 ' $q$ diminishes one period later, but increases two periods later. Additionally, Play 2, clusters $\mathrm{M}$ and $\mathrm{H}$ have positive effects on coordination, and also Session 1 distinguishes from Session 2 by increasing coordination. By interpreting the marginal effects per Plays 1 and 2, we only comment relevant differences: when player 1 changes his action from ' 1 ' to ' 0 ' $q$ always diminishes in Play 1, and Session 1 does not show differences from Session 2.

A positive marginal effect between the probability $p_{1}$ and nature indicates that player 1 follows nature. Nevertheless, the smarter players 1 (in cluster H) make signaling mistakes to inform at the cost of diminishing $p_{1}$. It is worth to comment that in Play 2 of Session 1, players 1 make more mistakes than in Session 2 as indicated by the negative marginal effect of dummy Session 1

Regarding probability $p_{2}$, it is remarkable the negative effect that Session 1 has on such probability, which might conceal over-signaling in Session 1. In contrast, clusters $\mathrm{M}$ and $\mathrm{H}$ have significant positive effect on the probability $p_{2}$.

Result 4: Some mis-signaling is observed in the behavior of player 1 subjects. When player 2 matches nature's action, player 1's and nature's actions match around the $98 \%$ of the times, on average. Whereas when player 2 does not match nature's action, player 1 matches nature's action significantly less in session 1 than in session 2 -around $25.99 \%$ less in session 1.

\section{Conclusions}

How efficiently players can coordinate under certain conditions in the lab is the scope of this paper. Specifically, our experimental set up has been inspired by the repeated matching pennies game with asymmetric information already introduced by Gossner et al. (2003, 2006). In their paper, Gossner et al. design optimal strategies of communication between sender and receiver in an infinitely repeated set-up. The authors use a binary information source and model the uncertainty coming from nature as a no-player playing a binomial random variable taking either the value 0 or 1 (each with probability 1/2). Players are characterized by their information available: the sender has private information on the future state of nature; while the receiver has public information about the history of nature's past states. The role of the strategic interaction is crucial since the gains of players are mutually conditional. Given that there is a positive gain when both players match nature's, the sender has an incentive to share information in order to improve his own gains. Thus, actions' 
coordination is possible being a strictly dominant strategy for both players. The main finding of that paper is the construction of optimal strategies based on communication blocks.

In order to create the correct experimental environment for testing the set-up described above, we first have determined that the length $n=55$ is the appropriate for the finite sequence to be generated randomly by nature. We have then provided a theoretical characterization for strategies designed and implemented under that experimental environment.

More specifically we have provided an information constraint with rational numbers that we call the rational information constraint. This constraint takes into consideration the fact that the number of bytes available for players to transmit information is finite. Furthermore, such a constraint is a necessary condition for communication to be possible but it is not sufficient, since an operational communication device should be implementable. To that purpose, we introduce as a refinement, the implementable information constraint, which is expressed in terms of combinatorial numbers. Therefore, this refinement takes into account the number of finite binary sequences under the requirements of the strategy of the two players.

We have implemented in the lab a specific channel for communication between players: previously to the playing of the game, a chat is activated during 3 minutes. During this time, players had the possibility to write free messages hopefully designing their strategies without explicit cost. How much information is transmitted depends on how informative the signal of player 1 is and how receptive player 2 is in receiving the signal. This will eventually determine the payoff of the two players. Here we have analysed the effectiveness of that chat on transmitting information in the terms of the theoretical model. That is, without specifically analysing what the messages in the chat contain, we have tested whether and how much did players coordinate under such conditions and, therefore, whether the model predicts reasonable strategies that could be observed from real heterogeneous agents.

Among our main experimental results we find that subjects design strategies at three levels of coordination. First, strategies at low level where the receiver ignores or misunderstands the sender's message, coordinating actions by pure chance. Second, a medium level of coordination in which strategies transmit successfully information by following a joint coordination code. A third level of coordination, the richest, where coordination codes achieve payoffs close to the optimal predicted by the theoretical model. Overall, we confirm that subjects coordinate their actions with experience, arriving to high levels of coordination in the second play of the session.

In a further analysis of the data, we have applied binary logit models. Theoreti- 
cally, actions of player 2 depend on nature's and player 1's behavior. Moreover, the full informed player 1 coordinates with the uninformed player 2 via a coordination strategies that are designed during the pre-play phase. Consequently, what player 1 does in the game depends only on nature's actions, this fact being common knowledge by the two players before the game starts. From our logit estimation of the matching probabilities we obtain that the behavior of player 2 subjects is significantly explained by actions taken by nature and the corresponding player 1. Moreover, player 1's action shift conveys nature's action mostly played. As far as player 1 subjects is concerned, there is somehow mis-signaling in the sense that, when player 2 matches nature, player 1 makes mistakes in excess thus deviating from the theoretical prediction. However, if we measure the deviations taking coordination clusters, we calculate that deviations are small, between $2 \%$ and $11 \%$. When player 2 does not match nature, we find opposite evidence from our data. In particular, in session 1 the estimated probability is much lower than the theoretical prediction, which can be interpreted as over-signaling, while in session 2 the contrary happens, which evidences under-signaling. Some mis-signaling is observed in the behavior of player 1 subjects.

Sumarising, we conclude that communication reduces inefficiencies and players follow a class of strategies, the block strategies, salient in the literature of repeated games in general and in Gossner et al. in particular. Finally, it is quite significant, that very little experience is enough to make a difference in the implementation by the part of informed players of strategies using mistakes, or strategic signaling, to convey information even in complex setups of dynamic environments under uncertainty. 


\section{References}

[1] Agastya M, Bag PK, Chakraborty I (2014) Communication and authority with a partially informed expert. The RAND Journal of Economics, 45(1):176-197.

[2] Agastya M, Bag PK, Chakraborty I (2015) Proximate preferences and almost full revelation in the CrawfordSobel game. Economic Theory Bulletin, 3(2):201-212.

[3] Allon G, Bassamboo A (2011) Buying from the babbling retailer? The impact of availability information on customer behavior. Management Science, 57(4):713726.

[4] Allon G, Bassamboo A, Gurvich I (2011) We will be right with you?: Managing customer expectations with vague promises and cheap talk. Operations Research, 59(6):1382-1394.

[5] Blume A, Ortmann A (2007) The effects of costless pre-play communication: Experimental evidence from games with Pareto-ranked equilibria. Journal of Economic Theory, 132:274-290.

[6] Crawford V, Sobel J (1982) Strategic information transmission. Econometrica, 50(6):1431-1451.

[7] Crawford V (1998) A survey of experiments on communication via cheap talk. Journal of Economic Theory, 78: 286-298.

[8] Devetag G, Ortmann A (2007) When and why? A critical survey on coordination failure in the laboratory. Experimental Economics, 10(3):331-344. https://doi.org/10.1007/s10683-007-9178-9

[9] Farrell J, Rabin M (1996) Cheap talk. Journal of Economic Perspectives 10(3):103-118.

[10] Gilligan TW, Krehbiel K (1989) Asymmetric information and legislative rules with a heterogeneous committee. American Journal of Political Science, 33:459490 .

[11] Gossner O, Hernández P, Neyman A (2003) Online matching pennies. Discussion Paper 316, The Hebrew University of Jerusalem.

[12] Gossner O, Hernández P, Neyman A (2006) Optimal use of communication resources. Econometrica, 74(6):1603-1636. 
[13] Hertel J, Smith J (2013) Not so cheap talk: Costly and discrete communication. Theory and Decision, 75(2):267-291.

[14] Kartik N (2005) Information transmission with almost-cheap talk. Technical report, www.najecon.org.

[15] Krishna V, Morgan J (2001) Asymmetric information and legislative rules: Some amendments. The American Political Science Review, 95(2):435-452.

[16] Sobel J (2010) Giving and receiving advise. Mimeo, University of California San Diego, presented at the Econometric Society 10th World Congress. 


\section{Appendix 1: Instructions of the experiment (trans- lated from Spanish)}

You are going to participate in an experimental session that will give you the possibility to earn some money in cash. How much money you will ultimately take will depend on luck and your and others' decisions. Please switch off your mobile phone and leave your things to one side. For your participation in the session you need just the instructions and the computer on your desk. Please raise your hand if you have any questions, and one of us will see to it privately.

In this experiment, you will be paired with another participant, who will not change throughout the session. A pair is composed of two types of participants: 'Type 1' and 'Type 2'. At the beginning of the session, the computer will randomly assign you a role and display it on your screen. The experiment is divided into two plays of 55 rounds each. At the beginning of each play, the computer will randomly determine, for every round, a value that may be either 0 or 1 . This value will be called 'Prize'. In each round, the probability that the Prize is associated to 0 or to 1 is exactly the same: $50 \%$ (it is like tossing a coin). Each of value will determine your earnings in each round, according to the following rules.

Each round, your decision making consists in choosing either 0 or 1 . In each pair, the two participants simultaneously choose either 0 or 1 taking into account that:

- If the decisions of both participants coincide with the Prize, they both get 1 ECU each in that round.

- If at least one decision within the pair does not coincide with Prize, then both get nothing in that round.

At the beginning of each block, you will have 3 minutes to communicate with your partner through a chat. You can end the chat at any time before the end by clicking on the option 'Exit from the chat'. Every message sent through the chat will be recorded and carefully analyzed by the those conducting the experiment. At the end of each round, your screen will display information concerning the value of the 'Prize' (0 or 1$)$, the decision of your partner (0 or 1$)$ and your own decision in that round.

To be 'Type 1' or 'Type 2' has consequences:

- If you are 'Type 1', at the beginning of each block of 55 rounds, and after using the chat to communicate with your partner, you will be aware of the sequence of values of the Prize that corresponds to that block.

- If you are 'Type 2', you will be aware of the value of the Prize at the end of each round. 
Moreover, participant 'the agent' knows that participant 'the wiser' will be aware of the values of Prize for each block just after the chat time. the wiser knows that the agent will have that information at the end of each round.

\section{Earnings}

At the end of each block, the participants in the experiment will know the number of winning rounds. At the end of the session, you will be paid your total payoff in cash, that is, the total number of rounds (in the two blocks of 55) in which you won the prize of 1 ECU. The exchange rate between ECUs and Euros is 1 ECU=1/4 Euro. 


\section{Appendix 2: Tables 6 and 7}

Table 4: Optimal strategies for blocks of length $m=\{2,3,4, \ldots, 27\}$ under a rational information constraint.

\begin{tabular}{|c|c|c|}
\hline$m$ & $\left(p^{*}, q^{*}\right)$ & $p^{*} q^{*}$ \\
\hline 2 & $\left(1, \frac{1}{2}\right),\left(\frac{1}{2}, 1\right)$ & $\frac{1}{2}$ \\
\hline 3 & $\left(1, \frac{2}{3}\right)$ & $\frac{2}{3}$ \\
\hline 4 & $\left(1, \frac{3}{4}\right)$ & $\frac{3}{4}$ \\
\hline 5 & $\left(1, \frac{3}{5}\right),\left(\frac{3}{4}, \frac{4}{5}\right)$ & $\frac{3}{5}$ \\
\hline 6 & $\left(1, \frac{4}{6}\right),\left(\frac{4}{5}, \frac{5}{6}\right)$ & $\frac{2}{3}$ \\
\hline 7 & $\left(1, \frac{5}{7}\right),\left(\frac{5}{6}, \frac{6}{7}\right)$ & $\frac{5}{7}$ \\
\hline 8 & $\left(1, \frac{6}{8}\right),\left(\frac{6}{7}, \frac{7}{8}\right)$ & $\frac{3}{4}$ \\
\hline 9 & $\left(\frac{7}{8}, \frac{8}{9}\right)$ & $\frac{7}{9}$ \\
\hline 10 & $\left(\frac{8}{9}, \frac{9}{10}\right)$ & $\frac{4}{5}$ \\
\hline 11 & $\left(1, \frac{8}{11}\right),\left(\frac{8}{9}, \frac{9}{11}\right),\left(\frac{8}{10}, \frac{10}{11}\right)$ & $\frac{8}{11}$ \\
\hline 12 & $\left(1, \frac{9}{12}\right),\left(\frac{9}{10}, \frac{10}{12}\right),\left(\frac{9}{11}, \frac{11}{12}\right)$ & $\frac{3}{4}$ \\
\hline 13 & $\left(1, \frac{10}{13}\right),\left(\frac{10}{11}, \frac{11}{13}\right),\left(\frac{10}{12}, \frac{12}{13}\right)$ & $\frac{10}{13}$ \\
\hline 14 & $\left(\frac{11}{12}, \frac{12}{14}\right),\left(\frac{11}{13}, \frac{13}{14}\right)$ & $\frac{11}{14}$ \\
\hline 15 & $\left(\frac{12}{13}, \frac{13}{15}\right)$ & $\frac{4}{5}$ \\
\hline 16 & $\left(1, \frac{12}{16}\right),\left(\frac{12}{13}, \frac{13}{16}\right),\left(\frac{12}{14}, \frac{14}{16}\right),\left(\frac{12}{15}, \frac{15}{16}\right)$ & $\frac{3}{4}$ \\
\hline 17 & $\left(1, \frac{13}{17}\right),\left(\frac{13}{14}, \frac{14}{17}\right),\left(\frac{13}{15}, \frac{15}{17}\right),\left(\frac{13}{16}, \frac{16}{17}\right)$ & $\frac{13}{17}$ \\
\hline 18 & $\left(\frac{14}{15}, \frac{15}{18}\right),\left(\frac{14}{16}, \frac{16}{18}\right),\left(\frac{14}{17}, \frac{17}{18}\right)$ & $\frac{7}{9}$ \\
\hline 19 & $\left(\frac{15}{16}, \frac{16}{19}\right),\left(\frac{15}{17}, \frac{17}{19}\right)$ & $\frac{15}{19}$ \\
\hline 20 & $\left(\frac{16}{17}, \frac{17}{20}\right),\left(\frac{16}{18}, \frac{18}{20}\right)$ & $\frac{4}{5}$ \\
\hline 21 & $\left(1, \frac{16}{21}\right),\left(\frac{16}{17}, \frac{17}{21}\right),\left(\frac{16}{18}, \frac{18}{21}\right),\left(\frac{16}{19}, \frac{19}{21}\right),\left(\frac{16}{20}, \frac{20}{21}\right)$ & $\frac{16}{21}$ \\
\hline 22 & $\left(1, \frac{17}{22}\right),\left(\frac{17}{18}, \frac{18}{22}\right),\left(\frac{17}{19}, \frac{19}{22}\right),\left(\frac{17}{20}, \frac{20}{22}\right)$ & $\frac{17}{22}$ \\
\hline 23 & $\left(\frac{18}{19}, \frac{19}{23}\right),\left(\frac{18}{20}, \frac{20}{23}\right),\left(\frac{18}{21}, \frac{21}{23}\right)$ & $\frac{18}{23}$ \\
\hline 24 & $\left(\frac{19}{20}, \frac{20}{24}\right),\left(\frac{19}{21}, \frac{21}{24}\right),\left(\frac{19}{22}, \frac{22}{24}\right)$ & $\frac{19}{24}$ \\
\hline 25 & $\left(\frac{20}{21}, \frac{21}{25}\right),\left(\frac{10}{11}, \frac{22}{25}\right)$ & $\frac{4}{5}$ \\
\hline 26 & $\left(\frac{21}{23}, \frac{23}{26}\right)$ & $\frac{21}{26}$ \\
\hline 27 & $\left(\frac{21}{22}, \frac{22}{27}\right),\left(\frac{21}{23}, \frac{23}{27}\right),\left(\frac{21}{24}, \frac{24}{27}\right),\left(\frac{21}{25}, \frac{25}{27}\right)$ & $\frac{7}{9}$ \\
\hline
\end{tabular}


Table 5: Optimal implementable strategies for sequences of length $n=\{5,6, \ldots, 60\}$.

\begin{tabular}{|c|c|c|c|c|c|c|c|c|}
\hline$n$ & $m^{*}$ & $p^{*}$ & $q^{*}$ & $p^{*} q^{*}$ & gain/block & $m^{*}\left(1-p^{*} q^{*}\right)$ & $n b\left(n, m^{*}\right)$ & total earnings \\
\hline 5 & 2 & 1 & $1 / 2$ & $1 / 2$ & 1 & 1 & 2 & 2 \\
\hline 6 & 2 & 1 & $1 / 2$ & $1 / 2$ & 1 & 1 & 2 & 2 \\
\hline 7 & 3 & 1 & $2 / 3$ & $2 / 3$ & 2 & 1 & 2 & 4 \\
\hline 8 & 3 & 1 & $2 / 3$ & $2 / 3$ & 2 & 1 & 2 & 4 \\
\hline 9 & 2 & 1 & $1 / 2$ & $1 / 2$ & 1 & 1 & 4 & 4 \\
\hline 9 & 3 & 1 & $2 / 3$ & $2 / 3$ & 2 & 1 & 2 & 4 \\
\hline 10 & 3 & 1 & $2 / 3$ & $2 / 3$ & 2 & 1 & 3 & 6 \\
\hline 11 & 3 & 1 & $2 / 3$ & $2 / 3$ & 2 & 1 & 3 & 6 \\
\hline 12 & 5 & $3 / 4$ & $4 / 5$ & $3 / 5$ & 3 & 2 & 2 & 6 \\
\hline 13 & 3 & 1 & $2 / 3$ & $2 / 3$ & 2 & 1 & 4 & 8 \\
\hline 14 & 3 & 1 & $2 / 3$ & $2 / 3$ & 2 & 1 & 4 & 8 \\
\hline 15 & 3 & 1 & $2 / 3$ & $2 / 3$ & 2 & 1 & 4 & 8 \\
\hline 16 & 3 & 1 & $2 / 3$ & $2 / 3$ & 2 & 1 & 5 & 10 \\
\hline 17 & 3 & 1 & $2 / 3$ & $2 / 3$ & 2 & 1 & 5 & 10 \\
\hline 18 & 3 & 1 & $2 / 3$ & $2 / 3$ & 2 & 1 & 5 & 10 \\
\hline 19 & 3 & 1 & $2 / 3$ & $2 / 3$ & 2 & 1 & 6 & 12 \\
\hline 20 & 3 & 1 & $2 / 3$ & $2 / 3$ & 2 & 1 & 6 & 12 \\
\hline 21 & 3 & 1 & $2 / 3$ & $2 / 3$ & 2 & 1 & 6 & 12 \\
\hline 22 & 3 & 1 & $2 / 3$ & $2 / 3$ & 2 & 1 & 7 & 14 \\
\hline 23 & 10 & $7 / 8$ & $4 / 5$ & $7 / 10$ & 7 & 3 & 2 & 14 \\
\hline 24 & 10 & $7 / 8$ & $4 / 5$ & $7 / 10$ & 7 & 3 & 2 & 14 \\
\hline 25 & 3 & 1 & $2 / 3$ & $2 / 3$ & 2 & 1 & 8 & 16 \\
\hline 26 & 3 & 1 & $2 / 3$ & $2 / 3$ & 2 & 1 & 8 & 16 \\
\hline 27 & 3 & 1 & $2 / 3$ & $2 / 3$ & 2 & 1 & 8 & 16 \\
\hline 28 & 3 & 1 & $2 / 3$ & $2 / 3$ & 2 & 1 & 9 & 18 \\
\hline 29 & 3 & 1 & $2 / 3$ & $2 / 3$ & 2 & 1 & 9 & 18 \\
\hline 30 & 13 & $9 / 10$ & $10 / 13$ & $9 / 13$ & 9 & 4 & 2 & 18 \\
\hline 31 & 3 & 1 & $2 / 3$ & $2 / 3$ & 2 & 1 & 10 & 20 \\
\hline 32 & 6 & $4 / 5$ & $5 / 6$ & $2 / 3$ & 4 & 2 & 5 & 20 \\
\hline 33 & 10 & $7 / 8$ & $4 / 5$ & $7 / 10$ & 7 & 3 & 3 & 21 \\
\hline 34 & 3 & 1 & $2 / 3$ & $2 / 3$ & 2 & 1 & 11 & 22 \\
\hline 35 & 3 & 1 & $2 / 3$ & $2 / 3$ & 2 & 1 & 11 & 22 \\
\hline 36 & 11 & $8 / 9$ & $9 / 11$ & $8 / 11$ & 8 & 3 & 3 & 24 \\
\hline 37 & 3 & 1 & $2 / 3$ & $2 / 3$ & 2 & 1 & 12 & 24 \\
\hline 38 & 3 & 1 & $2 / 3$ & $2 / 3$ & 2 & 1 & 12 & 24 \\
\hline 39 & 3 & 1 & $2 / 3$ & $2 / 3$ & 2 & 1 & 12 & 24 \\
\hline 40 & 3 & 1 & $2 / 3$ & $2 / 3$ & 2 & 1 & 13 & 26 \\
\hline 41 & 18 & $13 / 15$ & $5 / 6$ & $13 / 18$ & 13 & 5 & 2 & 26 \\
\hline 42 & 18 & $13 / 15$ & $5 / 6$ & $13 / 18$ & 13 & 5 & 2 & 26 \\
\hline 43 & 10 & $7 / 8$ & $4 / 5$ & $7 / 10$ & 7 & 3 & 4 & 28 \\
\hline 44 & 10 & $7 / 8$ & $4 / 5$ & $7 / 10$ & 7 & 3 & 4 & 28 \\
\hline 45 & 20 & $15 / 16$ & $4 / 5$ & $3 / 4$ & 15 & 5 & 2 & 30 \\
\hline 46 & 3 & 1 & $2 / 3$ & $2 / 3$ & 2 & 1 & 15 & 30 \\
\hline 47 & 11 & $8 / 9$ & $9 / 11$ & $8 / 11$ & 8 & 3 & 4 & 32 \\
\hline 48 & 11 & $8 / 9$ & $9 / 11$ & $8 / 11$ & 8 & 3 & 4 & 32 \\
\hline 49 & 15 & $11 / 12$ & $4 / 5$ & $11 / 15$ & 11 & 4 & 3 & 33 \\
\hline 50 & 15 & $11 / 12$ & $4 / 5$ & $11 / 15$ & 11 & 4 & 3 & 33 \\
\hline 51 & 15 & $11 / 12$ & $4 / 5$ & $11 / 15$ & 11 & 4 & 3 & 33 \\
\hline 52 & 3 & 1 & $2 / 3$ & $2 / 3$ & 2 & 1 & 17 & 34 \\
\hline 53 & 10 & $7 / 8$ & $4 / 5$ & $7 / 10$ & 7 & 3 & 5 & 35 \\
\hline 54 & 24 & $9 / 10$ & $5 / 6$ & $3 / 4$ & 18 & 6 & 2 & 36 \\
\hline 55 & 3 & 1 & $2 / 3$ & $2 / 3$ & 2 & 1 & 18 & 36 \\
\hline 56 & 25 & $19 / 20$ & $4 / 5$ & $19 / 25$ & 19 & 6 & 2 & 38 \\
\hline 57 & 25 & $19 / 20$ & $4 / 5$ & $19 / 25$ & 19 & 6 & 2 & 38 \\
\hline 58 & 11 & $8 / 9$ & $9 / 11$ & $8 / 11$ & 8 & 3 & 5 & 40 \\
\hline 59 & 11 & $8 / 9$ & $9 / 11$ & $8 / 11$ & 8 & 3 & 5 & 40 \\
\hline 60 & 11 & $8 / 9$ & $9 / 11$ & $8 / 11$ & 8 & 3 & 5 & 40 \\
\hline
\end{tabular}




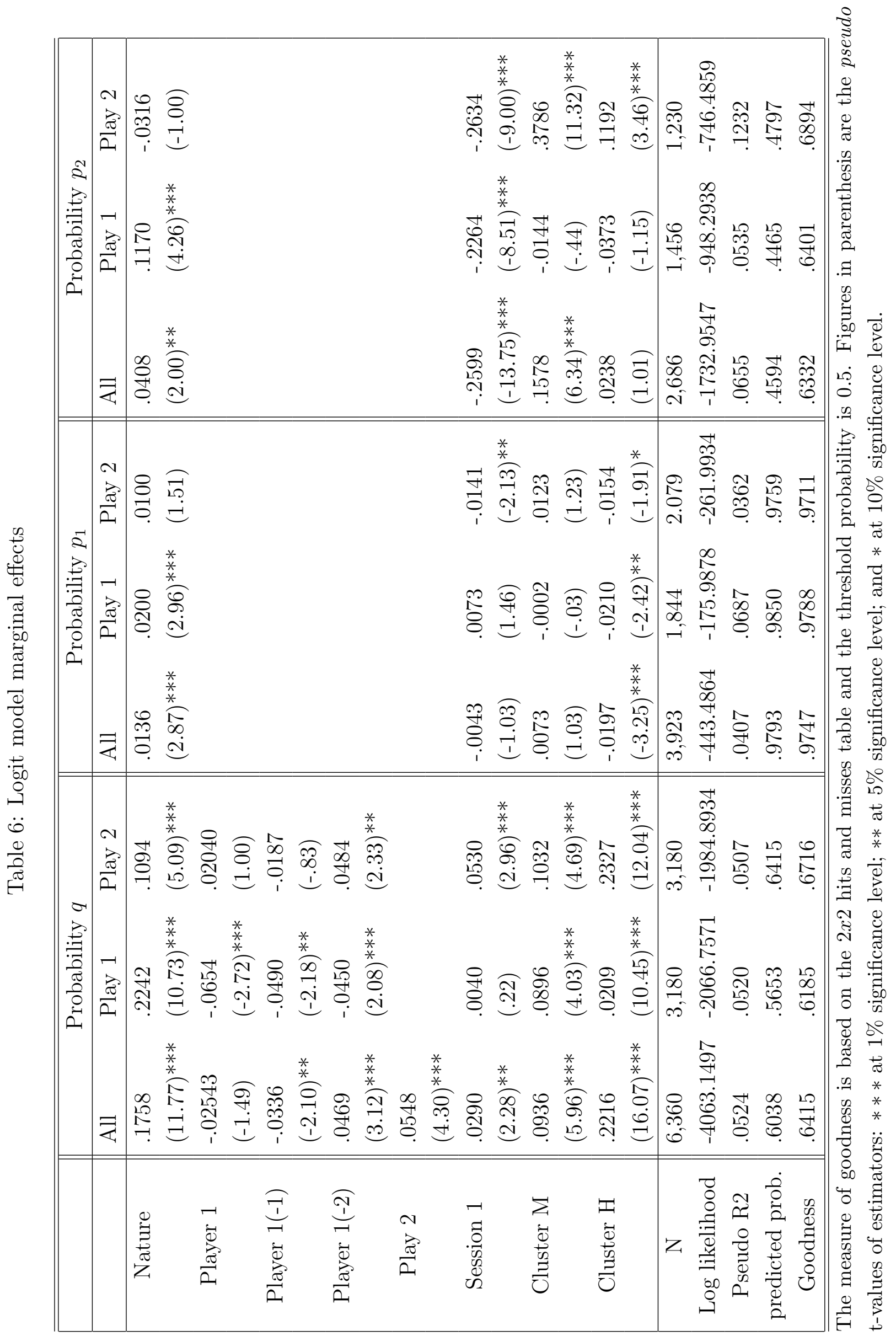

